\title{
Scheduling Problems and Generalized Graph Coloring
}

\author{
John Machacek \\ Department of Mathematics, Michigan State University, USA
}

\begin{abstract}
We define a new type of vertex coloring which generalizes vertex coloring in graphs, hypergraphs, and simplicial complexes. To this coloring there is an associated symmetric function in noncommuting variables for which we give a deletion-contraction formula. In the case of graphs our symmetric function in noncommuting variables agrees with the chromatic symmetric function in noncommuting variables of Gebhard and Sagan. Our vertex coloring is a special case of the scheduling problems defined by Breuer and Klivans. We show how the deletion-contraction law can be applied to scheduling problems.

Résumé. Nous définissons un nouveau type de coloration des sommets qui généralise les colorations dans les graphes, hypergraphes et complexes simpliciaux. Pour cette coloration, nous associons une fonction symétrique en variables non commutatives, pour laquelle nous donnons une formule de délétion - contraction. Dans le cas des graphes, notre fonction symétrique en variables non commutatives est en accord avec celle de Gebhard et Sagan. Notre coloration des sommets est un cas particulier des problémes d'ordonnancement définis par Breuer et Klivans; nous démontrons comment la loi de délétion - contraction peut être appliquée á ces problémes.
\end{abstract}

Keywords. coloring, symmetric functions, quasisymmetric functions

\section{Introduction}

In this paper we define a generalization of vertex coloring which has graph coloring and hypergraph coloring as a special case. Associated to our generalization vertex coloring we have a symmetric function in noncommuting variables which generalizes the chromatic symmetric function in noncommuting variables defined by Gebhard and Sagan in [GS01]. The vertex coloring we study corresponds to a special class of the scheduling problems defined by Breuer and Klivans in [BK14], and our symmetric function in noncommuting variables is an instance of the scheduling quasisymmetric function in noncommuting variables from [BK14].

\subsection{NCSym and NCQSym}

We let $\mathbb{P}=\{1,2, \ldots\}$ denote the positive integers and for any $n \in \mathbb{P}$ we let $[n]=\{1,2, \ldots, n\}$. A partition of $[n]$ is $\pi=B_{1} / B_{2} / \cdots / B_{l}$ where $\biguplus_{i=1}^{l} B_{i}=[n]$. When writing partitions we often suppress notation by simply writing $12 / 3$ in place of $\{1,2\} /\{3\}$. Here we call each $B_{i}$ a block of the partition $\pi$ and the order of the blocks is irrelevant. For example $12 / 3$ and $3 / 12$ denote the same partition of [3]. 
Take noncommuting variables $\left\{y_{1}, y_{2}, \ldots\right\}$ and a partition $\pi$ of $[n]$ for some $n \in \mathbb{P}$, then the monomial nc-symmetric function $m_{\pi}$ is defined by

$$
m_{\pi}:=\sum_{i_{1}, i_{2}, \ldots, i_{n}} y_{i_{1}} y_{i_{2}} \cdots y_{i_{n}}
$$

where the sum is over all sequences $\left(i_{1}, i_{2}, \ldots, i_{n}\right) \in \mathbb{P}^{n}$ satisfying the condition that $i_{j}=i_{k}$ if and only if $j$ and $k$ are in the same block of the partition $\pi$. Also, the powersum nc-symmetric function $p_{\pi}$ is defined by

$$
p_{\pi}:=\sum_{\sigma \geq \pi} m_{\sigma}
$$

where $\sigma \geq \pi$ is taken in the lattice of partitions of $[n]$ partially ordered by refinement. We denote the lattice of partitions of $[n]$ by $\Pi_{n}$. We now define NCSym the algebra of nc-symmetric functions to be the $\mathbb{K}$-space generated by either the basis of monomial nc-symmetric functions $\left\{m_{\pi}: \pi \in \Pi_{n}, n \in \mathbb{P}\right\}$ or the basis of powersum nc-symmetric functions $\left\{p_{\pi}: \pi \in \Pi_{n}, n \in \mathbb{P}\right\}$. Here $\mathbb{K}$ can be any field. As an example we have

$$
\begin{aligned}
m_{12 / 3} & =y_{1} y_{1} y_{2}+y_{2} y_{2} y_{1}+y_{1} y_{1} y_{3}+y_{3} y_{3} y_{1}+\cdots \\
p_{12 / 3} & =m_{12 / 3}+m_{123}
\end{aligned}
$$

as elements of NCSym.

A composition of $[n]$ is $\Phi=\left(B_{1}, B_{2}, \ldots, B_{l}\right)$ where $\biguplus_{i=1}^{l} B_{i}=[n]$. When writing composition we often suppress notation in the same manner as for partition by simply writing $(12,3)$ in place of $(\{1,2\},\{3\})$. We again call each $B_{i}$ a block of the composition $\Phi$. The only difference between in partition of $[n]$ and a composition of $[n]$ is in the latter the ordering of the blocks is relevant. For example $(12,3)$ and $(3,12)$ denote different compositions of [3]. Taking a composition $\Phi=\left(B_{1}, B_{2}, \ldots, B_{l}\right)$ of $[n]$ for some $n \in \mathbb{P}$ the monomial nc-quasisymmetric function is defined by

$$
M_{\Phi}:=\sum_{i_{1}, i_{2}, \ldots, i_{n}} y_{i_{1}} y_{i_{2}} \cdots y_{i_{n}}
$$

where the sum is over all sequences $\left(i_{1}, i_{2}, \ldots, i_{n}\right) \in \mathbb{P}^{n}$ satisfying the condition that for $j, k \in[n]$ where $j \in B_{p}$ and $k \in B_{q}$ with $p \leq q$ we have that

- $i_{j} \leq i_{k}$

- $i_{j}=i_{k}$ if and only if $p=q$.

We let $\Delta_{n}$ denote the collection of all compositions of $[n]$ and define NCQSym the algebra of $n c$ quasisymmetric functions to be the $\mathbb{K}$-space generated by the basis of monomial nc-quasisymmetric functions $\left\{M_{\Phi}: \Phi \in \Delta_{n}, n \in \mathbb{P}\right\}$. As an example we have

$$
\begin{aligned}
& M_{(12,3)}=y_{1} y_{1} y_{2}+y_{1} y_{1} y_{3}+y_{2} y_{2} y_{3}+\cdots \\
& M_{(3,12)}=y_{2} y_{2} y_{1}+y_{3} y_{3} y_{1}+y_{3} y_{3} y_{2}+\cdots
\end{aligned}
$$


as elements of NCQSym. We note that NCSym is contained in NCQSym as a proper subset. Given $\pi \in \Pi_{n}$ we have

$$
m_{\pi}=\sum_{\Phi} M_{\Phi}
$$

where the sum is over all compositions $\Phi$ of $[n]$ which have the same blocks as $\pi$. One can check $m_{12 / 3}=M_{(12,3)}+M_{(3,12)}$ using the previous examples in this section.

Given a monomial $y_{i_{1}} y_{i_{2}} \cdots y_{i_{n}}$ and a sequence $\left(r_{0}, r_{1}, \ldots, r_{k}\right) \in \mathbb{P}^{k+1}$ with $k<n$ we define induction on the monomial with respect to the sequence by

$$
y_{i_{1}} y_{i_{2}} \cdots y_{i_{n}} \uparrow\left(r_{0}, r_{1}, \ldots, r_{k}\right):=y_{i_{1}} y_{i_{2}} \cdots y_{i_{n-k}}^{1+r_{k}} \cdots y_{i_{n-1}}^{1+r_{1}} y_{i_{n}}^{1+r_{0}}
$$

Extending this operation linearly we get induction of any element of NCQSym, and hence any element of NCSym as well. Note this generalizes induction as defined in [GS01] where what we denote by $\uparrow^{(1)}$ is used. We can define induction on compositions in a way compatible with induction on NCQSym. Given a composition $\Phi \in \Delta_{n}, r \in \mathbb{P}$, and $t \in \mathbb{N}$ define $\Phi \uparrow^{s, t}$ to be the composition of $[n+s]$ obtained from $\Phi$ by first replacing $n-j$ with $n-j+s$ for $0 \leq j<t$ and then placing $n-t+1, n-t+2, \ldots, n-t+s$ in the same block as $n-t$. For $\left(r_{0}, r_{1}, \ldots, r_{k}\right) \in \mathbb{P}^{k+1}$ with $k<n$ we define $\Phi \uparrow\left(r_{0}, r_{1}, \ldots, r_{k}\right):=\Phi \uparrow\left(r_{0}, r_{1}, \ldots, r_{k}\right), 0$ where we have the recursion $\Phi \uparrow\left(r_{0}, r_{1}, \ldots, r_{j}\right), t=\left(\Phi \uparrow r_{0}, t\right) \uparrow\left(r_{1}, r_{2}, \ldots, r_{j}\right), t+r_{0}+1$ with $\Phi \uparrow(), t=\Phi$.

Lemma 1. If $\Phi \in \Delta_{n}$ and $\left(r_{0}, r_{1}, \ldots, r_{k}\right) \in \mathbb{P}^{k+1}$ with $k<n$, then $M_{\Phi} \uparrow\left(r_{0}, r_{1}, \ldots, r_{k}\right)=M_{\Phi \uparrow\left(r_{0}, r_{1}, \ldots, r_{k}\right)}$.

The lemma follows from the definition of the induction operation. Induction can be applied to partitions in the same way as compositions but without regrading the ordering of the blocks. Lemma 1 then implies that

$$
m_{\pi} \uparrow\left(r_{0}, r_{1}, \ldots, r_{k}\right)=m_{\pi \uparrow\left(r_{0}, r_{1}, \ldots, r_{k}\right)} \quad p_{\pi} \uparrow\left(r_{0}, r_{1}, \ldots, r_{k}\right)=p_{\pi \uparrow\left(r_{0}, r_{1}, \ldots, r_{k}\right)}
$$

whenever $\pi \in \Pi_{n}$ and $k<n$. We now demonstrate the induction operation with an example.

Example 2. We take $(1,2) \in \Delta_{2}$ and $(2,1) \in \mathbb{P}^{2}$. First let us consider induction on the composition $(1,2)$.

$$
\begin{aligned}
(1,2) \uparrow^{(2,1)} & =(1,2) \uparrow(2,1), 0 \\
& =(1,234) \uparrow(1), 3 \\
& =(12,345)
\end{aligned}
$$

Next we consider induction on the nc-monomial quasisymmetric function and see that is in compatible with induction on the composition.

$$
\begin{aligned}
M_{(1,2)} & =y_{1} y_{2}+y_{1} y_{3}+y_{2} y_{3}+\cdots \\
M_{(1,2)} \uparrow^{(2,1)} & =y_{1}^{2} y_{2}^{3}+y_{1}^{2} y_{3}^{3}+y_{2}^{2} y_{3}^{3}+\cdots \\
M_{(1,2) \uparrow(2,1)} & =M_{(12,345)}=y_{1}^{2} y_{2}^{3}+y_{1}^{2} y_{3}^{3}+y_{2}^{2} y_{3}^{3}+\cdots
\end{aligned}
$$




\subsection{Scheduling Problems}

As defined in [BK14] a scheduling problem on $n$ elements is a boolean formula $S$ over the atomic formulas $x_{i} \leq x_{j}$ for $i, j \in[n]$. We are interested in solutions to a scheduling problem where each $x_{i}$ takes a value in $\mathbb{P}$. A function $f:[n] \rightarrow \mathbb{P}$ is a solution to the scheduling problem $S$ if when $x_{i}=f(i)$ the boolean formula $S$ is true. We then get the scheduling nc-quasisymmetric function $\mathcal{S}_{S}$ defined by

$$
\mathcal{S}_{S}:=\sum_{f} \prod_{i=1}^{n} y_{f(i)}
$$

where the sum is taken over all solutions $f$ to the scheduling problem $S$. Given $\Phi=\left(B_{1}, B_{2}, \ldots, B_{l}\right)$ a composition of $[n]$ we can view $\Phi$ as a map $\Phi:[n] \rightarrow[l]$ by $f(i)=j$ if $i \in B_{j}$. We say a set composition $\Phi$ solves $S$ its corresponding map does. In this way we see that $\mathcal{S}_{S}$ is indeed an element of NCQSym and can be expressed in the monomial basis as

$$
\mathcal{S}_{S}=\sum_{\Phi} M_{\Phi}
$$

where the sum in over set compositions $\Phi$ of $[n]$ which solve $S$.

\subsection{Coloring in Graphs, Hypergraphs, and Simplicial Complexes}

For us a graph is will mean a finite undirected graph with loops and multiple edges allowed. We will write a graph $G$ as a pair $G=(V, E)$ where where $V$ is a finite set and $E$ is finite multiset of unordered pairs of (not necessarily distinct) elements of $V$. We call elements of $V$ vertices and elements of $E$ edges. When $|V|=n$ we will usually assume without stating that $V=[n]$. From identifying $V$ with $[n]$ we obtain an ordering of the vertices. Given vertices $u, v \in V$, the edge between $u$ and $v$ is written $u v \in E$ where $u v=v u$. A map $f: V \rightarrow \mathbb{P}$ is called a proper coloring of $G$ if it produces no monochromatic edge. That is $f$ is a proper coloring if for all $u v \in E$ we have that $f(u) \neq f(v)$.

A hypergraph $H$ is a pair $H=(V, E)$ where $E$ a collection of subsets of $V$. We call the elements of $V$ vertices and elements of of $E$ hyperedges. If for each $e \in E$ we have that $|e|=s$, then we call $H$ an $s$-uniform hypergraph. A map $f: V \rightarrow \mathbb{P}$ is a proper coloring of $H$ if it produces no monochromatic hyperedge.

An abstract simplicial complex $\Gamma$ on a vertex set $V$ is a collection of subsets of $V$ such that for all $v \in V$ we have $\{v\} \in \Gamma$ and if $A \in \Gamma$ then $B \in \Gamma$ for any $B \subseteq A$. Elements of $\Gamma$ are called faces and faces which are maximal with respect to inclusion are called facets. We call $A \in \Gamma$ an $s$-simplex if $|A|=s+1$. Given a positive integer $s$ a map $f: V \rightarrow \mathbb{P}$ is an $s$-simplicial coloring of $\Gamma$ if it produces no monochromatic $s$-simplex. Coloring in graphs and hypergraphs is classical, but this notion of coloring in simplicial complexes is more recent and defined in [DMN10]. We will show in Section 4 that coloring in simplicial complexes can be thought of as coloring in uniform hypergraphs and conversely.

\section{Coloring and Generalized Graphs}

We define a generalized graph $\mathcal{G}$ to be a pair $\mathcal{G}=(V, \mathcal{E})$ where $V$ is a finite set and $\mathcal{E}$ is a multiset of nonempty graphs with vertex set $V$. This means elements of $\mathcal{E}$ are of the form $(V, E)$ with $E \neq \emptyset$. An element $(V, E) \in \mathcal{E}$ is called a generalized edge, and $(V, E) \in \mathcal{E}$ is a generalized loop if $E$ consists of 
only loops. If $\mathcal{G}=(V, \mathcal{E})$ is a generalized graph, then a coloring of $\mathcal{G}$ is a map $f: V \rightarrow \mathbb{P}$. The coloring $f: V \rightarrow \mathbb{P}$ is a proper coloring of $\mathcal{G}$ if for each $(V, E) \in \mathcal{E}$ there exists an edge $e \in E$ which is not monochromatic. Observe that no proper color of $\mathcal{G}$ exists if $\mathcal{G}$ contains a generalized loop.

Example 3. Given a graph $G=(V, E)$ we get a generalized graph $\mathcal{G}_{G}:=\left(V, \mathcal{E}_{G}\right)$ where $\mathcal{E}_{G}=$ $\{(V,\{e\}): e \in E\}$. Here proper colorings of $\mathcal{G}_{G}$ exactly correspond to proper colorings of $G$. For a hypergraph $H=(V, E)$ we get the generalized graph $\mathcal{G}_{H}:=\left(V, \mathcal{E}_{H}\right)$ where $\mathcal{E}_{H}=\left\{\left(V, E_{e}\right): e \in E\right\}$ where $E_{e}=\{u v: u, v \in e\}$. Again here with have that the proper colorings of $\mathcal{G}_{H}$ are in correspondence with proper colorings of $H$.

We will see in Lemma 16 that $s$-simplicial coloring is equivalent to proper coloring $(s+1)$-uniform hypergraphs. Therefore considering Example 3 coloring in generalized graphs encompasses coloring in graphs, hypergraphs, and simplicial complexes.

For a generalized graph $\mathcal{G}$ we define the chromatic nc-symmetric function of $\mathcal{G}$ by

$$
Y_{\mathcal{G}}:=\sum_{f} \prod_{i=1}^{n} y_{f(i)}
$$

where the sum is over all proper colorings $f$ of $\mathcal{G}$. It is readily verified that $Y_{\mathcal{G}}$ is in fact an element of NCSym. By allowing the variables to commute we obtain the symmetric function $X_{\mathcal{G}}$ which we call the chromatic symmetric function of $\mathcal{G}$. We also obtain the chromatic polynomial of $\mathcal{G}$, which we denote $\chi_{\mathcal{G}}$, by letting $\chi_{\mathcal{G}}(k)$ by the specialization of $Y_{\mathcal{G}}$ with $y_{i}=1$ for $1 \leq i \leq k$ and $y_{i}=0$ for $i>k$. Here $\chi_{\mathcal{G}}(k)$ counts the number of proper coloring of $\mathcal{G}$ using only colors from $[k]$.

For a generalized graph $\mathcal{G}=(V, \mathcal{E})$ and $(V, E) \in \mathcal{E}$ deletion of the generalized edge $(V, E)$ is denoted $\mathcal{G} \backslash(V, E)$ and defined by

$$
\mathcal{G} \backslash(V, E):=\left(V, \mathcal{E}^{\prime}\right)
$$

where $\mathcal{E}^{\prime}=\mathcal{E} \backslash(V, E)$. For any graphs $\left(V, E_{1}\right)$ and $\left(V, E_{2}\right)$ on the same vertex set we define

$$
\left(V, E_{1}\right) /\left(V, E_{2}\right):=\left(V /\left(V, E_{2}\right), E_{1} /\left(V, E_{2}\right)\right)
$$

where $V /\left(V, E_{2}\right)$ and $E_{1} /\left(V, E_{2}\right)$ are obtained by identifying the vertices $u$ and $v$ whenever $u v \in E_{2}$. For a generalized graph $\mathcal{G}=(V, \mathcal{E})$ and $(V, E) \in \mathcal{E}$ contraction by the generalized edge $(V, E)$ is denoted by $\mathcal{G} /(V, E)$ and defined by

$$
\mathcal{G} /(V, E)=\left(V /(V, E), \mathcal{E}^{\prime \prime}\right)
$$

where $\mathcal{E}^{\prime \prime}=\left\{G /(V, E): G \in \mathcal{E}^{\prime}\right\}$ and again $\mathcal{E}^{\prime}=\mathcal{E} \backslash(V, E)$. Observe that these definitions agree with the usual notion of deletion and contraction in a graph $G$ if we consider the generalized graph $\mathcal{G}_{G}$ from Example 3 Also, note that for a generalized loop deletion and contraction are equivalent. Lastly, notice that both deletion and contraction always decrease the number of generalized edges by exactly 1 .

Let $\mathcal{G}=(V, \mathcal{E})$ be a generalized graph and let $(V, E) \in \mathcal{E}$ be a fixed generalized edge. We call the generalized edge $(V, E)$ contraction ready if the blocks of the partition of $V$ given by connected components of the graph $(V, E)$ can be ordered to obtain the composition $\Phi=\left(B_{1}, B_{2}, \ldots, B_{l}\right)$ of $V$ such that $B_{i_{1}}>B_{i_{2}}$ for any $i_{1}<i_{2}$ and there is some $k \geq 0$ such that $B_{i}$ a singleton if and only if $i>k$. In this case we call $\Phi$ a contraction ready composition. Here for disjoint subsets $A, B \subseteq V$ we say $A<B$ if $a<b$ for all $a \in A$ and $b \in B$. Notice that any generalized edge can be made contraction ready by some relabeling of the vertices. Here if $|V|=n$ we identify $V$ with $[n]$, and relabeling the vertices 
amounts to acting by some permutation $\delta \in S_{n}$. The permutation $\delta$ also acts on a monomial of degree $n$ by

$$
\delta\left(y_{i_{1}} y_{i_{2}} \cdots y_{i_{n}}\right)=y_{i_{\delta^{-1}(1)}} y_{i_{\delta-1}(2)} \cdots y_{i_{\delta-1}(n)}
$$

and can be extended linearly to act on $Y_{\mathcal{G}}$. In this case we have $\delta\left(Y_{\mathcal{G}}\right)=Y_{\delta(\mathcal{G})}$. For the corresponding relabeling result for graphs see [GS01, Proposition 3.3]. Thus we can assume that any generalized graph $\mathcal{G}$ has a contraction ready generalized edge since we can always obtain such a generalized edge by relabeling.

If $(V, E)$ is a contraction ready generalized edge then the contraction operation is compatible with our ordering of vertices. When the contraction ready composition $\Phi=\left(B_{1}, B_{2}, \ldots, B_{l}\right)$ the $l$ blocks the contraction $\mathcal{G} /(V, E)$ will have vertex set $[l]$ where the vertices in $B_{i}$ all get identified to a single vertex denoted by $l-i+1$. To demonstrate this consider the following example.

Example 4. Consider the generalized graph $\mathcal{G}=([4],\{([4],\{13,24\}),([4],\{12,34\})\})$ and let $G=$ $([4],\{12,34\})$. Then $\mathcal{G} \backslash G=([4],\{([4],\{13,24\})\})$ and $\mathcal{G} / G=([2],\{([2],\{12,12\})\})$. Here the contraction ready composition is $(34,12)$. The vertices 3 and 4 are identified and denote by 2 while the vertices 1 and 2 are identified and denoted by 1 in $\mathcal{G} / G$. An example of a proper coloring of $\mathcal{G}$ is given by $f:[4] \rightarrow \mathbb{P}$ by $f(1)=f(2)=f(3)=1$ and $f(4)=2$.

We are now ready to state and prove the deletion-contraction formula for the chromatic nc-symmetric function of a generalized graph.

Theorem 5. If $\mathcal{G}=(V, \mathcal{E})$ and $(V, E) \in \mathcal{E}$ is a contraction ready generalized edge, then

$$
Y_{\mathcal{G}}=Y_{\mathcal{G} \backslash(V, E)}-Y_{\mathcal{G} /(V, E)} \uparrow^{\left(r_{1}, r_{2}, \ldots, r_{k}\right)}
$$

where $\Phi=\left(B_{1}, B_{2}, \ldots, B_{l}\right)$ is the contraction ready composition of $V$ given by connected components of $(V, E)$ and $r_{i}=\left|B_{i}\right|-1$.

Proof. Given $f: V \rightarrow \mathbb{P}$ observe that $f$ being monochromatic on all edges $e \in E$ is equivalent to being $f$ monochromatic on each connected component of $(V, E)$. Now we have

$$
Y_{\mathcal{G} \backslash(V, E)}=\sum_{f} \prod_{i=1}^{n} y_{f(i)}
$$

where the sum is over proper colorings of $\mathcal{G} \backslash(V, E)$. Any proper coloring of $\mathcal{G} \backslash(V, E)$ is of one of the following two types.

- A proper coloring of $\mathcal{G}$.

- A proper coloring of $\mathcal{G} \backslash(V, E)$ which is monochromatic on each connected component of $(V, E)$.

Then by the definition on contraction, induction, and the fact the $(V, E)$ was contraction ready we have that

$$
Y_{\mathcal{G} /(V, E)} \uparrow^{\left(r_{1}, r_{2}, \ldots, r_{k}\right)}=\sum_{f} \prod_{i=1}^{n} y_{f(i)}
$$

where the sum is over $f: V \rightarrow \mathbb{P}$ such that $f$ is a proper coloring of $\mathcal{G} \backslash(V, E)$ which $f$ is monochromatic on each component of $(V, E)$. The theorem readily follows. 
Our deletion-contraction law in Theorem 5 generalizes the deletion-contraction law in [GS01], and in a similar way we can use our deletion-contract law to give a powersum expansion of the chromatic ncsymmetric function $Y_{\mathcal{G}}$. Given a generalized graph $\mathcal{G}=(V, \mathcal{E})$ and $A \subseteq \mathcal{E}$ with $A=\left\{\left(V, E_{i}\right): 1 \leq i \leq\right.$ $k\}$ we define $\pi(A)$ to be the partition of $V$ into the connected components of the graph $\left(V, \bigcup_{i=1}^{k} E_{i}\right)$.

Theorem 6. If $\mathcal{G}=(V, \mathcal{E})$ is a generalized graph, then

$$
Y_{\mathcal{G}}=\sum_{A \subseteq \mathcal{E}}(-1)^{|A|} p_{\pi(A)}
$$

Proof. We induct on $|\mathcal{E}|$. If $|\mathcal{E}|=0$, then $Y_{\mathcal{G}}=p_{1 / 2 / \cdots / n}=p_{\pi(\emptyset)}$ and the theorem holds. Now assume $|\mathcal{E}|>0$ and let $(V, E) \in \mathcal{E}$ be a contraction ready generalized edge. As before we let $\mathcal{E}^{\prime}$ and $\mathcal{E}^{\prime \prime}$ denote the multiset of generalized edges in the deletion and contraction respectively. Then using Theorem 5 we have

$$
\begin{aligned}
Y_{\mathcal{G}} & =Y_{\mathcal{G} \backslash(V, E)}-Y_{\mathcal{G} /(V, E)} \uparrow^{\left(r_{1}, r_{2}, \ldots, r_{k}\right)} \\
& =\sum_{A \subseteq \mathcal{E}^{\prime}}(-1)^{|A|} p_{\pi(A)}-\sum_{\bar{A} \subseteq \mathcal{E}^{\prime \prime}}(-1)^{|\bar{A}|} p_{\pi(\bar{A})} \uparrow\left(r_{1}, r_{2}, \ldots, r_{k}\right) \\
& =\sum_{A \subseteq \mathcal{E}:(V, E) \notin A}(-1)^{|A|} p_{\pi(A)}+\sum_{A \subseteq \mathcal{E}:(V, E) \in A}(-1)^{|A|} p_{\pi(A)} \\
& =\sum_{A \subseteq \mathcal{E}}(-1)^{|A|} p_{\pi(A)} .
\end{aligned}
$$

We note that

$$
-\sum_{\bar{A} \subseteq \mathcal{E}^{\prime \prime}}(-1)^{|\bar{A}|} p_{\pi(\bar{A})} \uparrow^{\left(r_{1}, r_{2}, \ldots, r_{k}\right)}=\sum_{B \subseteq \mathcal{E}:(V, E) \in B}(-1)^{|B|} p_{\pi(B)}
$$

since for $\bar{A} \subseteq \mathcal{E}^{\prime \prime}$ we have $\pi(\bar{A}) \uparrow\left(r_{1}, r_{2}, \ldots, r_{k}\right)=\pi(B)$ where $B=A \cup(V, E)$ if $A \in \mathcal{E}^{\prime}$ corresponds to $\bar{A} \in \mathcal{E}^{\prime \prime}$. Recall $\mathcal{E}^{\prime \prime}=\mathcal{E}^{\prime} /(V, E)$ and hence we have a canonical bijection between $\mathcal{E}^{\prime}$ and $\mathcal{E}^{\prime \prime}$.

Allowing the variables to commute we obtain powersum expansion of the chromatic symmetric function $X_{\mathcal{G}}$ of generalized graph $\mathcal{G}$ from Theorem 6 . When considering graphs and hypergraphs as generalized graphs as in Example 3 we recover the powersum expansion for graphs [Sta95] and hypergraphs [Sta98]. Also, Lemma 16 will allow Theorem 6 to be applied in the setting of simplicial complexes we get a powersum expansion for the $s$-chromatic symmetric function from [BHM15]. We now conclude this section with an example of using both Theorem 5 and Theorem 6 to compute a chromatic nc-symmetric function.

Example 7. Again consider the generalized graph $\mathcal{G}=([4],\{([4],\{13,24\}),([4],\{12,34\})\})$ and let $G=([4],\{12,34\})$. Recall that $\mathcal{G} \backslash G=([4],\{([4],\{13,24\})\})$ and $\mathcal{G} / G=([2],\{([2],\{12,12\})\})$. Note $f:[4] \rightarrow \mathbb{P}$ is a proper coloring of $\mathcal{G}$ except in the following cases:

- $f(1)=f(2)$ and $f(3)=f(4)$

- $f(1)=f(3)$ and $f(2)=f(4)$. 
It follows that

$$
Y_{\mathcal{G}}=\sum_{\substack{\pi \neq 1234 \\ \pi \neq 12 / 34 \\ \pi \neq 13 / 24}} m_{\pi}
$$

Similarly by considering proper colorings of $\mathcal{G} \backslash G$ and $\mathcal{G} / G$ we can conclude

$$
Y_{\mathcal{G} \backslash G}=\sum_{\substack{\pi \neq 1234 \\ \pi \neq 12 / 34}} m_{\pi} \quad Y_{\mathcal{G} / G}=m_{1 / 2} \quad Y_{\mathcal{G} / G} \uparrow^{(1,1)}=m_{12 / 34}
$$

We can now directly verify Theorem 5 in this case which states $Y_{\mathcal{G}}=Y_{\mathcal{G} \backslash G}-Y_{\mathcal{G} / G} \uparrow^{(1,1)}$. We can alternatively use Theorem 6 to compute and obtain

$$
Y_{\mathcal{G}}=p_{1 / 2 / 3 / 4}-p_{12 / 34}-p_{13 / 24}+p_{1234} .
$$

It is readily verified that the expansions of $Y_{\mathcal{G}}$ in the monomial basis and powersum basis describe the same element of NCSym.

\section{Graph-like Scheduling Problems}

Though the definition of scheduling problems only includes atomic formulas with weak inequalities we can build strict inequality, equality, and nonequality as follows

$$
\left(x_{i}<x_{j}\right)=\neg\left(x_{j} \leq x_{i}\right) \quad\left(x_{i}=x_{j}\right)=\left(x_{i} \leq x_{j}\right) \wedge\left(x_{j} \leq x_{i}\right) \quad\left(x_{i} \neq x_{j}\right)=\neg\left(x_{i}=x_{j}\right) .
$$

A boolean formula $C$ is called edge-like if it can be expressed as a disjunction of nonequalities. That is $C$ is edge-like if

$$
C=\bigvee_{(i, j) \in I} x_{i} \neq x_{j}
$$

for some $I \subseteq[n] \times[n]$. A boolean formula $S$ is called graph-like if it can be expressed as a conjunction of edge-like boolean formulas. That is $S$ is graph-like if

$$
S=\bigwedge_{\alpha \in I} C_{\alpha}
$$

for some index set $I$ where $C_{\alpha}$ is edge-like for each $\alpha \in I$.

Example 8. For a generalized graph $\mathcal{G}=(V, \mathcal{E})$ define the scheduling problem $S_{\mathcal{G}}$ by

$$
S_{\mathcal{G}}:=\bigwedge_{(V, E) \in \mathcal{E}} \bigvee_{u v \in E} x_{u} \neq x_{v}
$$

Here $S_{\mathcal{G}}$ is the scheduling problem of properly coloring $\mathcal{G}$ where $x_{v}$ represents the color given to $v \in V$.

Our next result shows that any graph-like scheduling problem is equivalent to properly coloring some generalized graph, and hence for any graph-like scheduling problem the scheduling nc-quasisymmetric function is the chromatic nc-symmetric function for some generalized graph. In particular $\mathcal{S}_{S}$ lies in NCSym whenever $S$ is graph-like. The fact $\mathcal{S}_{S}$ is nc-symmetric when $S$ is graph-like is not surprising and also follows from the fact that $x_{i} \neq x_{j}$ is symmetric in $i$ and $j$. 
Theorem 9. A scheduling problem $S$ is graph-like if and only if $S=S_{\mathcal{G}}$ for some generalized graph $\mathcal{G}$.

Proof. Let $S$ be a graph-like scheduling problem where $S=C_{1} \wedge C_{2} \wedge \cdots \wedge C_{m}$ with each $C_{i}$ edge-like, and

$$
C_{i}=\bigvee_{(j, k) \in I_{i}} x_{j} \neq x_{k}
$$

for some $I_{i} \subseteq[n] \times[n]$. We define the generalized graph $\mathcal{G}=([n], \mathcal{E})$ where $\mathcal{E}=\left\{E_{1}, E_{2}, \ldots, E_{m}\right\}$ and $E_{i}=\left\{j k:(j, k) \in I_{i}\right\}$. We then have $S=S_{\mathcal{G}}$. The reverse direction is shown in Example 8

Theorem 9 has the following immediate corollary.

Corollary 10. If $S$ is a graph-like scheduling problem, then $\mathcal{S}_{S}=Y_{\mathcal{G}}$ for some generalized graph $\mathcal{G}$.

Let $S$ be any scheduling problem and let $V(S)$ be the set of $i$ such that $x_{i}$ appears in $S$. If $C$ is edgelike, then $C=\bigvee_{(i, j) \in I} x_{i} \neq x_{j}$ for some $I \subseteq[n] \times[n]$. Thus $\neg C=\bigwedge_{(i, j) \in I} x_{i}=x_{j}$ and we say that $i \sim_{C} j$ if $x_{i}=x_{j}$ appears in $\neg C$. We get an equivalence relation on $V(C)$ by taking the reflexive transitive closure of $\sim_{C}$. We will write $V(C)=O_{1} \uplus O_{2} \uplus \cdots \uplus O_{k}$ to denote the decomposition of $V(C)$ into $\sim_{C}$ equivalence classes.

We define a contraction operation on scheduling problems. Let $S$ be a boolean formula over the atomic formulas $x_{i} \leq x_{j}$ for $i, j \in[n]$. Given a positive integers $r$ and $t$ with $r+t<n$ we define $S \downarrow_{r, t}$ to be the boolean formula over atomic formulas $x_{i} \leq x_{j}$ for $i, j \in[n-r]$ obtained from $S$ where $x_{n-t}, x_{n-t-1}, \ldots, x_{n-t-r}$ are all identified to $x_{n-r-t}$ and then variable indices are standardized to lie in $[n-r]$. For $\left(r_{1}, r_{2}, \ldots, r_{k}\right)$ a sequence of positive integers with $r=\sum_{i=1}^{k} r_{i}$ and $r+k<n$. We define $S \downarrow\left(r_{1}, r_{2}, \ldots, r_{k}\right):=S \downarrow\left(r_{1}, r_{2}, \ldots, r_{k}\right), 0$ where we have the recursion $S \downarrow\left(r_{1}, r_{2}, \ldots, r_{j}\right), t=$ $\left(S \downarrow_{r_{1}, t}\right) \downarrow_{\left(r_{2}, r_{3}, \ldots, r_{j}\right), t+1}$ with $S \downarrow(), t=S$. We now give an example of contraction of a scheduling problem.

\section{Example 11.}

$$
\begin{aligned}
& \left(\left(x_{1} \leq x_{2}\right) \vee\left(x_{1}<x_{3}\right) \vee\left(x_{2} \neq x_{4}\right) \vee\left(x_{4} \leq x_{5}\right)\right) \downarrow^{(2,1)} \\
= & \left(\left(x_{1} \leq x_{2}\right) \vee\left(x_{1}<x_{3}\right) \vee\left(x_{2} \neq x_{4}\right) \vee\left(x_{4} \leq x_{5}\right)\right) \downarrow^{(2,1), 0} \\
= & \left(\left(x_{1} \leq x_{2}\right) \vee\left(x_{1}<x_{3}\right) \vee\left(x_{2} \neq x_{3}\right) \vee\left(x_{3} \leq x_{3}\right)\right) \downarrow^{(1), 1} \\
= & \left(\left(x_{1} \leq x_{1}\right) \vee\left(x_{1}<x_{2}\right) \vee\left(x_{1} \neq x_{2}\right) \vee\left(x_{2} \leq x_{2}\right)\right)
\end{aligned}
$$

Lemma 12. If $S=S^{\prime} \wedge C$ is scheduling problem, then $\mathcal{S}_{S}=\mathcal{S}_{S^{\prime}}-\mathcal{S}_{S^{\prime} \wedge \neg C}$.

Proof. We need to just consider which set compositions which solve $S$. We observe that $\mathcal{S}_{S}$ and $\mathcal{S}_{S^{\prime}}-$ $\mathcal{S}_{S^{\prime} \wedge \neg C}$ are both sums of monomials $M_{\Phi}$ such that $\Phi$ solves $S$.

Recall for two disjoint subsets $A, B \subseteq[n]$ we say $A<B$ if $a<b$ for all $a \in A$ and $b \in B$. Given a scheduling problem $S$ on $n$ elements with $S=S^{\prime} \wedge C$ we call $C$ a contractible clause if:

- $C$ is edge-like,

- $V(C)=\left\{x_{n-s}, x_{n-s+1}, \ldots, x_{n}\right\}$ for some $0<s \leq n$,

- $O_{i}>O_{j}$ for $i<j$ where $V(C)=O_{1} \uplus O_{2} \uplus \cdots \uplus O_{k}$ and the $O_{i}$ are the $\sim_{C}$ equivalence classes. 
Note that any edge-like clause $C$ can be made into a contractible clause by relabeling the variables $x_{i}$ if needed. The relabeling works similarly to the relabeling for generalized graphs in the previous section.

Lemma 13. If $S=S^{\prime} \wedge C$ is a scheduling problem and $C$ is a contractible clause, then

$$
\mathcal{S}_{S^{\prime} \wedge \neg C}=\left(\mathcal{S}_{S^{\prime} \downarrow\left(r_{1}, r_{2}, \ldots, r_{k}\right)}\right) \uparrow^{\left(r_{1}, r_{2}, \ldots, r_{k}\right)}
$$

where $V(C)=O_{1} \uplus O_{2} \uplus \cdots \uplus O_{k}$ is the decomposition into $\sim_{C}$ equivalence classes and $r_{i}=\left|O_{i}\right|-1$.

Proof. First note that $\mathcal{S}_{S^{\prime} \wedge \neg C}$ is the sum over monomials $M_{\Phi}$ such that $\Phi$ solves $S^{\prime}$ and $\neg C$. That is $\Phi$ solves $S^{\prime}$ and $i$ and $j$ are in the same block whenever $x_{i} \sim_{C} x_{j}$.

Given any $\Phi$ solving $S^{\prime} \downarrow\left(r_{1}, r_{2}, \ldots, r_{k}\right)$ we need to verify that $\Phi \uparrow\left(r_{1}, r_{2}, \ldots, r_{k}\right)$ solves $S^{\prime}$ and that $i$ and $j$ are in the same block whenever $x_{i} \sim_{C} x_{j}$. Both these conditions are satisfied by definition of contraction and induction along with the fact that $C$ is a contractible clause.

We now give a deletion-contraction law that applies to any scheduling problem $S$ that can be expressed at $S=S^{\prime} \wedge C$ where $C$ is edge-like. The deletion-contraction law follows immediately from Lemma 12 and Lemma 13 .

Theorem 14. If $S=S^{\prime} \wedge C$ if a scheduling problem and $C$ is a contractible clause, then

$$
\mathcal{S}_{S}=\mathcal{S}_{S^{\prime}}-\left(\mathcal{S}_{S^{\prime} \downarrow\left(r_{1}, r_{2}, \ldots, r_{k}\right)}\right) \uparrow^{\left(r_{1}, r_{2}, \ldots, r_{k}\right)}
$$

where $V(C)=O_{1} \uplus O_{2} \uplus \cdots \uplus O_{k}$ is the decomposition into $\sim_{C}$ equivalence classes and $r_{i}=\left|O_{i}\right|-1$.

If $S=C_{1} \wedge C_{2} \wedge \cdots \wedge C_{m}$ is graph-like with each $C_{i}$ edge-like, then we can apply Theorem 14 with $S^{\prime}=C_{1} \wedge C_{2} \wedge \cdots \wedge C_{m-1}$ and $C=C_{m}$. In this case both $S^{\prime}$ and $S^{\prime} \downarrow_{\left(r_{1}, r_{2}, \ldots, r_{k}\right)}$ are graph-like and so Theorem 14 can be iterated (after perhaps relabeling). If $S$ is a graph-like scheduling problem, then by Theorem 9 we have that $S=S_{\mathcal{G}}$ for a generalized graph $\mathcal{G}$. In that case the deletion-contraction for $\mathcal{S}_{S}$ in Theorem 14 is the same as the deletion-contraction for $Y_{\mathcal{G}}$ in Theorem 5 . We now given an example of using Theorem 14 to compute a scheduling nc-quasisymmetric function.

Example 15. We let $S=S^{\prime} \wedge C$ where

$$
S^{\prime}=\left(x_{1} \leq x_{2}\right) \wedge\left(x_{2} \leq x_{3}\right) \wedge\left(x_{3} \leq x_{4}\right) \quad C=\left(x_{1} \neq x_{2}\right) \vee\left(x_{3} \neq x_{4}\right) .
$$

We then let

$$
S^{\prime \prime}=S^{\prime} \downarrow_{(1,1)}=\left(x_{1} \leq x_{1}\right) \wedge\left(x_{1} \leq x_{2}\right) \wedge\left(x_{2} \leq x_{2}\right)=\left(x_{1} \leq x_{2}\right) .
$$

Computing the scheduling nc-quasisymmetric functions we see

$$
\begin{aligned}
\mathcal{S}_{S^{\prime}} & =M_{(1234)}+M_{(1,234)}+M_{(12,34)}+M_{(123,4)}+M_{(1,2,34)}+M_{(1,23,4)}+M_{(12,3,4)}+M_{(1,2,3,4)} \\
\mathcal{S}_{S^{\prime \prime}} & =M_{(12)}+M_{(1,2)} \\
\mathcal{S}_{S^{\prime \prime}} \uparrow^{(1,1)} & =M_{(1234)}+M_{(12,34)} .
\end{aligned}
$$

We then can apply Theorem 14

$$
\begin{aligned}
\mathcal{S}_{S} & =\mathcal{S}_{S^{\prime}}-\mathcal{S}_{S^{\prime \prime}} \uparrow(1,1) \\
& =M_{(1,234)}+M_{(123,4)}+M_{(1,2,34)}+M_{(1,23,4)}+M_{(12,3,4)}+M_{(1,2,3,4)} .
\end{aligned}
$$




\section{Coloring Uniform Hypergraphs and Simplicial Complexes}

In this section we first show that coloring in a simplicial complex can be thought of as coloring in a uniform hypergraph. Given a simplicial complex $\Gamma$ and a nonnegative integer $s$ we get an $(s+1)$-uniform hypergraph $H^{(s)}(\Gamma)=\left(V(\Gamma), E^{(s)}(\Gamma)\right)$ with edge set defined by

$$
E^{(s)}(\Gamma):=\{A \in \Gamma:|A|=s+1\} .
$$

Lemma 16. A map $f$ is an s-simplicial coloring of a simplicial complex $\Gamma$ if and only if $f$ is a proper coloring of $H^{(s)}(\Gamma)$.

Since we have seen in Example 3 that hypergraph coloring is a special case of coloring in generalized graphs, Lemma 16 shows that coloring is simplicial complexes is also a special case of generalized graph coloring. Thus the theory of the chromatic nc-symmetric function applies to simplicial complexes. Given a simplicial complex $\Gamma$ and positive integer $s$ the function $Y_{\mathcal{G}_{H^{(s)}}}$ is a noncommutative analogue for the $s$-chromatic symmetric function studied in [BHM15].

Next we show that coloring in a uniform hypergraph can be thought of as an instance of coloring in a simplicial complex. Given any $H=(V, E)$ we get a simplicial complex $\Gamma(H)$ on the vertex set $V$ defined by

$$
\Gamma(H)=\{A: A \subseteq e \in E\} \cup\{\{v\}: v \in V\} .
$$

Lemma 17. A map $f$ is a proper coloring of an $(s+1)$-uniform hypergraph $H$ if and only if $f$ is an $s$-simplicial coloring of $\Gamma(H)$.

Note a simplicial complex is determined completely by its facets, and we can rephrase the property of being an $s$-simplicial coloring in terms of facets. For a simplicial complex $\Gamma$ with vertex set $V$ a map $f: V \rightarrow \mathbb{P}$ is an $s$-simplicial coloring if and only if each facet of $\Gamma$ contains at most $s$ vertices of a given color. If $H$ is an $(s+1)$-uniform hypergraph, then $\Gamma(H)$ is the simplicial complex with facets given by the hyperedges of $H$ along with possibly a some isolated vertices. In particular, the simplicial complex $\Gamma(H)$ can be obtained from $H$ in linear time.

We define the decision problem $(k, s)$-SIMPLICIAL COLORABLE which takes as input a simplicial complex $\Gamma$ and outputs true if and only if $\Gamma$ can be $s$-simplicial colored using at most $k$ colors. Similarly we define the decision problem $(k, s)$-COLORABLE which takes as input a $s$-uniform hypergraph $H$ and outputs true if and only if $H$ can be properly colored using at most $k$ colors. Thus Lemma 17 says by considering $H \mapsto \Gamma(H)$ we have a polynomial time reduction from $(k, s+1)$-COLORABLE to $(k, s)$-SIMPLICIAL COLORABLE. We get the following proposition which addresses the question NPcompleteness of simplicial coloring asked in [BHM15]. We use the fact that $(k, s)$-COLORABLE is NPcomplete unless $k=1, s=1$, or $(k, s)=(2,2)$ [Lov73].

Proposition 18. For $k=1$ or $(k, s)=(2,1)$ we have $(k, s)$-SIMPlicial COLORABLE $\in \mathbf{P}$ and for all other pairs $(k, s)$ we have that $(k, s)$-SIMPLICIAL COLORABLE is NP-complete.

Proof. We certainly have the $(k, s)$-Simplicial COlORABle $\in \mathbf{N P}$ for any $(k, s)$. Note the if $s=1$ we are considering graph coloring and if $k=1$ we simply need to determine the dimension of the simplicial complex. The $\mathbf{N P}$-hardness for $k \neq 1$ and $(k, s) \neq(2,1)$ follows from the polynomial time reduction from $(k, s+1)$-COLORABLE by $H \mapsto \Gamma(H)$. 


\section{References}

[BHM15] C. Benedetti, J. Hallam, and J. Machacek. Combinatorial Hopf algebras of simplicial complexes. arXiv:1505.04458v1, 2015.

[BK14] F. Breuer and C. J. Klivans. Scheduling problems. arXiv:1401.2978v1, 2014.

[DMN10] N. Dobrinskaya, J. M. Møller, and D. Notbohm. Vertex colorings of simplicial complexes. arXiv:1007.0710v1, 2010.

[GS01] D. Gebhard and B. Sagan. A chromatic symmetric function in noncommuting variables. $J$. Alg. Combin., 13:227-255, 2001.

[Lov73] L. Lovász. Coverings and colorings of hypergraphs. In Proceedings of the Fourth Southeastern Conference on Combinatorics, Graph Theory, and Computing, pages 3-12, 1973.

[Sta95] Richard P. Stanley. A symmetric function generalization of the chromatic polynomial of a graph. Advances in Math., 111:166-194, 1995.

[Sta98] R. P. Stanley. Graphs colorings and related symmetric functions: Ideas and applications. Discrete Math., 193:267-286, 1998. 